\title{
miR-144/451 cluster plays an oncogenic role in esophageal cancer by inhibiting cell invasion
}

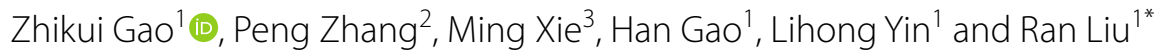

\begin{abstract}
Background: miRNA clusters are widely expressed across species, accumulating evidence has illustrated that miRNA cluster functioned more efficiently than single miRNA in cancer oncogenesis. It is likely that miRNA clusters are more stable and reliable than individual miRNA to be biomarkers for diagnosis and therapy. We previously found low expression of miR-144/451 was closely related with the risk for esophageal cancer. Researches on miR-144/451 cluster were mostly focused on individual miRNA but not the whole cluster, the regulatory mechanism of miRNA cluster were largely unknown.
\end{abstract}

Methods: In present study, we firstly analysed biological functions of individual miRNAs of miR-144/451 in ECa9706 transfected with miRNA mimics. We further analysed the biological function of the whole cluster in stable transgenic cell overexpressing miR-144/451. We then performed genome-wide mRNA microarray to detect differentially expressed gene profiles in stable transgenic cells.

Results: Overexpression of miR-144-3p promoted early apoptosis of ECa9706 and inhibited cell migration, cell invasion and cell proliferation. miR-144-5p and miR-451a inhibited cell proliferation, at the same time, miR-451a inhibited cell migration. Overexpression of miR-144/451 leads to the arrest cell cycle from S to G2 and G2 to M,while the invasion ability was obviously inhibited. We further observed c-Myc, p-ERK were downregulated in cells overexpressing miR-144/451, while p53 was up-regulated. The downstream effectors of c-Myc, MMP9 and p-cdc2 were downregulated in miR-144/451 stable transgenic cell. miR-144/451 may or partly inhibited cell cycles and invasion of ECa9706 through inhibiting ERK/c-Myc signaling pathway.

Conclusion: Collectively, we analysed the function of miR-144/451 cluster from individual to overall level. miR144/451 cluster played proto oncogene role in esophageal cancer by inhibiting cell invasion.

Keywords: Esophageal cancer, miRNA cluster, miR-144-3p, miR-144-5p, miR-451a

\section{Background}

Esophageal cancer is a common digestive system cancer with high incidence and mortality. According to statistics of WHO, esophageal cancer ranks as the fourth highest cause of cancer-related mortality in the world, there are 456,000 new cases and 400,000 deaths in 2012, more than half of the new cases occurs in China [1]. It is worth

\footnotetext{
*Correspondence: ranliu@seu.edu.cn

${ }^{1}$ Key Laboratory of Environmental Medicine Engineering, Ministry of Education, School of Public Health, Southeast University, Nanjing 210009, China

Full list of author information is available at the end of the article
}

mentioning the incidence is still growing. Although endoscopic resection is effective for esophageal cancer patients at early stage, the overall 5-year survival rate is still no more than $20 \%[2,3]$. There are no effective treatment for advanced esophageal cancer, so the key point for raising the survival rate of esophageal cancer is detecting and treating at early stage [4]. However, no obvious clinical symptom for esophageal cancer can be observed, searching for sensitive and specific biomarkers for early esophageal cancer becomes especially important.

Growing evidence showed that miRNAs played important roles in post transcriptional regulation by binding to 
the $3^{\prime} \mathrm{UTR}$ region of mRNAs, directing the repression of protein expression [5]. Abnormal miRNA-mediated regulation can affect oncellular functions which were closely related to the occurrence of tumor, miRNAs seem to be important molecular markers in the diagnosis of tumor $[6,7]$. Usually, miRNA genes were transcribed under the regulation of promoter and operon, in particular some were closely aligned on chromosome formed as a cluster. These clusters are suspected to transcribe together, though not exclusively, mediate synergistic or antagonistic regulatory effects. miRNA cluster is likely to be more stable and reliable than individual miRNA to be biomarkers for diagnosis $[8,9]$. However, most of the studies on miRNA clusters focused on individual miRNAs but not the cluster, regulatory mechanisms of miRNA cluster were largely unknown.

miR-144/451 cluster is highly conversed in different species, miRbase database (http://www.mirba se.org/) shows miR-144/451 cluster is constituted by miR-144-3p, miR-144-5p, miR-451a, miR-4732-3p and miR-4732-5p (Table 1). In previous study, we discovered the low-expression of miR-144/451 was closely related with the risk for esophageal cancer [10]. miR-144-3p was reported to be related with gastric carcinoma, lung cancer, hepatoma and colorectal cancer [11-14], miR144-5p was reported to be related with bladder cancer and colorectal cancer $[15,16]$, miR-451a was reported to be related with lung cancer, gastric carcinoma, colorectal cancer, liver cancer, breast cancer and osteosarcoma [17-22]. However, few is known about the function and mechanism of miR-144/451 in esophageal cancer. Most of the researches about miR-144/451 is focused only on single miRNAs but not on the cluster.

In present study, we analysed functions of individual miRNA of miR-144/451 in ECa9706, we established stable transgenic cells overexpressing miR-144/451 and analysed the function of the cluster. To better understand the potential mechanism of the cluster, genome-wide mRNA microarray and Western blot were performed. Results

Table 1 Location and sequences of miR-144/451 cluster

\begin{tabular}{lll}
\hline miRNA & Sequence & Location (GRCh38) \\
\hline miR-144-3p & $\begin{array}{c}\text { UACAGUAUAGAUGAUGUA } \\
\text { CU }\end{array}$ & chr17: 28861584-28861603 \\
miR-144-5p & $\begin{array}{c}\text { GGAUAUCAUCAUAUACUG } \\
\text { UAAG }\end{array}$ & chr17: 28861547-28861568 \\
miR-451a & AAACCGUUACCAUUACUG & chr17: 28861385-28861406 \\
& AGUU & \\
miR-4732-3p & GCCCUGACCUGUCCUGUU & chr17: 28861663-28861685 \\
miR-4732-5p & UGUAGAGCAGGGAGCAGG & chr17: 28861697-28861717 \\
& AAGCU & \\
\hline
\end{tabular}

of the present study may advance our understanding of the expression pattern and functional role of the miR144/451 cluster in esophageal carcinoma.

\section{Materials and methods \\ Cell line}

ECa9706, ECa109, H5E46, Het-1A and HEK-293T cells were provided by Key Laboratory of Environmental Medicine Engineering, Ministry of Education, School of Public Health, Southeast University. Cells were grown in RPMI-1640 containing 10\% fetal bovine serum (Gibico), $100 \mathrm{U} / \mathrm{mL}$ penicillin-streptomycin solution (Gibico) at $37{ }^{\circ} \mathrm{C}$ in incubator containing $5 \% \mathrm{CO}_{2}$ humidified atmosphere. Transient transfected Cells over-expressing miRNAs were obtained using micrON ${ }^{\mathrm{TM}}$ miRNA mimic (RiboBio). Lipofectamine ${ }^{\circledR}$ RNAiMAX Reagent (Invitrogen) were used for transfection according to manufacturer's instructions. Stable expression cell strain over-expressing miR-144/451 cluster were obtained using lentiviral transfection.

\section{Plasmids construction, virus production, and infection of target cells}

To obtain pri-miR-144/451 cluster plasmid, a synthetic sequence within the range of upstream $200 \mathrm{bp}$ to downstream 200 bp of pri-miR-144/451 was cloned into PCDH-CMV-MCS-EF1-Puro lentivirus plasmid. Plasmids of pMDLg/pRRE, pRSV-Rev, pMD2G and PCDHCMV-MCS-EF1-Puro were transfected into HEK-293T for packaging lentivirus.

\section{Antibodies and reagents}

PTEN (138G6) Rabbit Monoclonal antibody, Phosphocdc2 (Tyr15) antibody, Phospho-p44/42 MAPK (ERK1/2) (Thr202/Tyr204) Rabbit Monoclonal antibody, p44/42 MAPK (Erk1/2) Rabbit Monoclonal antibody, Phospho$\beta$-Catenin (Ser33/37/Thr41) antibody, Total- $\beta$-Catenin antibody, Phospho-c-Myc (Ser62) Rabbit Monoclonal antibody, p53 Rabbit Monoclonal antibody, Non-phospho (Active) $\beta$-Catenin (Ser33/37/Thr41) Rabbit Monoclonal antibody were obtained from Cell Signaling Technology (CST). Mouse anti-human C-myc monoclonal antibody, anti-MMP-9 antibody were obtained from Millipore.

\section{Apoptosis and cell cycle}

For cells transfected with mimics, cell apoptosis were detected using Annexin V-APC/7-AAD Apoptosis Detection Kit (KGA1026, KeyGEN BioTECH) according to the manufacture's protocol, for cells stably transfected with Lentiviral vectors, cell apoptosis was detected using (KGA1026, KeyGEN BioTECH). Flow cytometry (PI staining) were used for detecting cell cycle using PI 
cell cycle Detection Kit (KGA107, KeyGEN BioTECH) according to the manufacture's protocol, experiments were done in triplicates.

\section{Cell proliferation assay}

Cell proliferation were detected using 5-ethynyl-2'deoxyuridine (EdU) labeling/detection kit (Ribobio). Firstly, cells with a density of $1 \times 104$ cells/well were planted on 96-well plates, after 24 h, $50 \mathrm{mM}$ EdU were added into the plate for an additional $2 \mathrm{~h}$. Then the cells were fixed with $4 \%$ formaldehyde in PBS for $30 \mathrm{~min}$ and incubate with glycine for $5 \mathrm{~min}$. After washing with PBS and $0.5 \%$ TritonX-100 in PBS, cells were incubated with $1 \times$ Apollo dye at room temperature in dankness for $30 \mathrm{~min}$. At last, wash cells with $0.5 \%$ TritonX-100 in PBS and methanol, incubate cells with $1 \times$ Hoechst 33342 dye at room temperature in dankness for $30 \mathrm{~min}$. Preserve labeled cells in PBS. Observe and photograph using fluorescence microscopy, select five images randomly for cell counting, Assays were performed with five parallels.

\section{Invasion and migration}

Cell invasion and migration were detected using $8.0 \mu \mathrm{m}$ Transwell chamber (Corning). For cell migration, $5 \times 10^{4}$ cells were seeded into the upper chamber in serum-free media, the lower chamber were filled with RPMI-1640 containing $10 \%$ fetal bovine serum, culture for $24 \mathrm{~h}$, color and count migrated cell. For cell invasion, $5 \times 10^{5}$ cells were seeded into the upper chamber using serumfree media, the lower chamber were filled with RPMI1640 containing $50 \%$ fetal bovine serum, culture for $24 \mathrm{~h}$, color and count invasive cells, experiments were done in triplicates.

\section{RNA extraction and genome-wide mRNA microarray}

Total RNA were isolated using Trizol reagent (Invitrogen). RNA concentration were measured using Nanodrop 2000 (Thermo Fisher) and Agilent 2100 Bioanalyzer (Agilent). aRNA were obtained using GeneChip 3'IVT PLUS Kit (Affymetrix). mRNA profiles were detected using Affymetrix GeneChip primeview human Expression Array (100 format) in miR-144/451 overexpressing and negative control cells, experiments were done in triplicates.

Bioinformatics analysis of microarray and target prediction Ingenuity Pathway Analysis (IPA) were performed for analysing mRNA profiles detected by genome-wide mRNA microarray.

\section{Western blot analysis}

Cellular protein were extracted with cold RIPA buffer (Beyotime) containing protease and phosphatase inhibitors (Millipore). Lysates were cleared by centrifugation at $14,000 \mathrm{rpm}$ at $4{ }^{\circ} \mathrm{C}$ for $15 \mathrm{~min}$. Protein concentration were detected using bicinchoninic acid (BCA) assay (Thermo Fisher). Aliquots of protein $(20 \mu \mathrm{g})$ were separated by $10 \%$ SDS-PAGE and the separated proteins were transferred onto $0.45 \mu \mathrm{m}$ PVDF membrane (Millipore). Membranes were blocked with $5 \%(\mathrm{w} / \mathrm{v})$ non-fat milk in Tris- $\mathrm{HCl}$ buffered saline ( $\mathrm{pH}$ 7.4) with Tween-20 and incubated with the primary antibody overnight at $4{ }^{\circ} \mathrm{C}$. Subsequently, membranes were washed with Tris- $\mathrm{HCl}$ buffered saline and incubated with secondary antibody conjugated to horseradish peroxidase respectively diluted in 1:1000, at room temperature for $1 \mathrm{~h}$. Membranes were washed in Tris- $\mathrm{HCl}$ buffered saline and bounds were detected with SuperSignal West Femto/Pico Kit (Thermo Fisher). Blots were visualized and quantified using Tanon-5200 Imaging System (Tanon).

\section{RT-QPCR}

All primers (Bulge-Loop ${ }^{\mathrm{TM}}$ miRNA RT-QPCR Primer kits) for miRNA were purchased from Guangzhou RiboBio Co., Ltd. All primers for mRNA were synthetized in GenScript Corporation, U6 was selected as internal reference for miRNAs. The Sequence for forward primer of pri-miRNA was ACAGTGCTTTTCAAGCCATGC, for reverse primer, the sequence was GGGTGCCCGGAC TAGTACAT. $\beta$-actin was selected as internal reference for detecting pri-miRNA, sequence for forward primer was: ATCCGCAAAGACCTGT, and for reverse primer the sequence was: GGGTGTAACGCAACTAAG.

Total RNA $(\sim 2 \mu \mathrm{g})$ were extracted using Trizol regent (Invitrogen). cDNA was synthesized using Moloney Murine Leukemia Virus (MMLV) reverse transcriptase (Promega) and ribonuclease inhibitor (Thermo Fisher). SYBR Green mastermix were purchased from Toyobo Technologies. QPCR reactions were performed using StepOnePlus system (Applied Biosystems). Data for miRNA and mRNA were normalized using U6 and $\beta$-actin respectively. Expression of miRNA and mRNA were presented as relative RNA expression using $\Delta \Delta C_{T}$ formula (the fold change in target gene expression was equal to $2^{-\Delta \Delta \mathrm{CT}}$ ). All results were presented as mean of triplicates \pm SD of three independent experiments.

\section{Statistical analysis}

Statistical analysis was performed using SPSS 17.0. Group differences were explored by Student's t-test and analysis of variance (ANOVA). $P$ value $<0.05$ was considered to be statistically significant. 


\section{Results}

\section{Expression of miR-144/451 in different cells}

We detected the expression of miR-144/451 in ECa9706, ECa109, H5E46 and Het-1A. miR-144-3p, miR-144-5p, miR-451a and miR-4732-3p were low expressed (Fig. 1a), Eca9706 were selected for further experiment.

\section{Transfection efficiency of miRNA mimics}

In ECa9706, miRNA mimics significantly up-regulated expression of miR-144-3p, miR-144-5p, miR-451a, miR4732-3p and miR-4732-5p. Fold changes for all these five miRNAs were more than 1000.

\section{Effects of individual miRNAs on biological function}

miR-144-3p decreased the proportion of cells in G2 phase, but in cells over-expressing miR-451a, the proportion of cells in G2 increased. In cells overexpressing miR-144-3p and miR-451a there are (14.62 \pm 1.41$) \%$ and $(22.84 \pm 0.97) \%$ cells in G2 phase respectively, while in control the proportion is $(18.20 \pm 1.12) \%$ (Fig. 1b). miR-144-3p significantly promoted cell apoptosis with the early apoptosis rate of $(18.70 \pm 2.11) \%$, while in control, the early apoptosis rate was $(9.00 \pm 1.15) \%$ (Fig. 1c). Proliferation rate of cells overexpressing miR144-3p, miR-144-5p, miR-451a and miR-4732-3p were $(34.18 \pm 5.83) \%, \quad(33.56 \pm 3.94) \%, \quad(34.15 \pm 2.94) \%$ and $(31.50 \pm 2.01) \%$ respectively, in control the proliferation rate was $(41.16 \pm 2.13) \%$ (Fig. 1d). For cell migration, compared with the number of migrated cells of $36.67 \pm 3.58$ in control, miR-144-3p, miR-451a, miR4732-3p and miR-4732-5p inhibited cell migration, the number of cells passed through the membrane were $15.63 \pm 1.00,21.27 \pm 1.70,26.97 \pm 3.47$ and $24.87 \pm 1.36$ respectively. No difference were observed between cells over-expressing miR-144-5p and the control (Fig. 1e). For cell invasion, miR-144-3p obviously inhibited invasive ability, only $12.07 \pm 1.10$ cells passed through reconstituted basement membrane in cells overexpressing miR133-3p, while the number was $25.90 \pm 2.26$ in control. For cell proliferation, miR-144-3p, miR-144-5p, miR-451a and miR-4732-3p significantly inhibited proliferation of ECa9706 (Fig. 1e).

\section{Expression of miR-144/451 cluster in stable cell line}

In cells overexpressing pri-miR-144/451, miR-144-3p, miR-144-5p and miR-451a were significantly up-regulated, the fold change were 152.22, 699.41 and 600.49 respectively. No significant difference of the expression of miR-4732-3p and miR-4732-5p were found between pri-miR-144/451 and the control. miR-4732-3p and miR4732-5p seems not to be the member of miR-144/451 cluster (Table 2).

\section{Effects of miR-144/451 on biological function}

Overexpression of miR-144/451 increased the proportion of cells in phase of G2 and S, thus cells in G1 apparently reduced (Fig. 2a). No difference of apoptosis rate between cell over-expressing miR-144/451 and the control cell were observed (Fig. 2b). Overexpression of miR-144/451 have no effect on cell proliferation and migration (Fig. 2c, d). Transwell assay showed overexpression of miR$144 / 451$ obviously inhibited cell invasion, the inhibition rate reached $50 \%$ (Fig. $2 \mathrm{~d}$ ).

\section{Effects of miR-144/451 on gene expression}

According to the standard of $\mid$ Fold change $\mid \geq 1.3$ and $P$-value $<0.05$, there are 17 up-regulated and 57 downregulated genes in cells overexpressing miR-144/451 were detected (Fig. 3a, b), detailed data were shown in Additional file 1: Table S1.

\section{Possible upstream regulators of the differently expressed mRNAs}

IPA were used for analysing the possible upstream regulators, TGFB1, TNF, MAPK1, ERK, TP53, P38 MAPK, SMAD3, TCF/LEF, MMP1, EGFR, MMP2, WNT5A were predicted to be possible upstream regulators, detailed data were shown in Additional file 2: Table S2. According to the predicted interaction of moleculars, we predicted possible networks, the most enriched network were given as Fig. 3c.

\section{Effect of miR-144-451 gene cluster on key protein}

Stable transgenic and miRNA mimic transfected cells were collected individually. Protein expression of selected cells were detected using Western Blot. The expression of protein was evaluated by integrated option density (IOD), Relative expression were evaluated using the ratio of target protein and internal reference protein (Additional file 3: Table S3). Overexpression of miR-144/451 and individual miRNA have no effect on expression of total and unphosphorylated $\beta$-catenin. miR-144-3p, miR-144-5p, miR-451a, miR-4732-3p and miR-4732-5p slightly increased the expression of phosphorylated

(See figure on next page.)

Fig. 1 Effects of overexpression of single miRNA on cell functions. a Effects of overexpression of sigle miRNA on cell cycle. $\mathbf{b}$ Effects of overexpression of single miRNA on cell apoptosis. $\mathbf{c}$ Effects of overexpression of single miRNA on cell proliferation. $\mathbf{d}$ Effects of overexpression of single miRNA on cell migration and invasion 

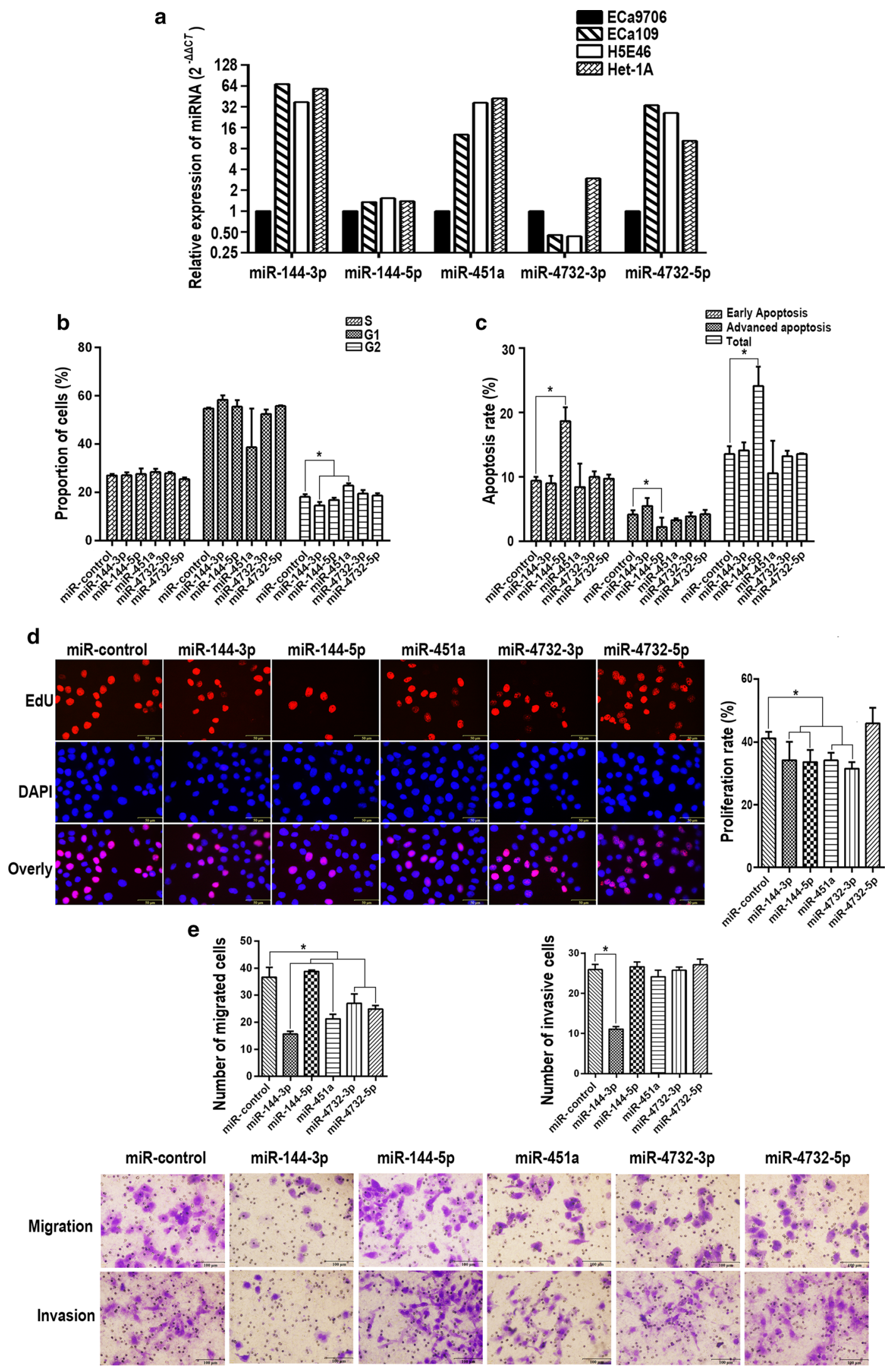
Table 2 Expression of miR-144/451 in cells overexpressing pri-miR-144/451

\begin{tabular}{llllll}
\hline miRNA & $\Delta C_{T}$ & $\Delta \Delta C_{T}$ & $\underset{\Delta \Delta C T}{2^{-}}$ & $P$ value \\
\cline { 2 - 3 } & Mimic & Control & & & \\
\hline
\end{tabular}

\begin{tabular}{llllll}
\hline miR-144-3p & $19.65 \pm 0.46$ & $26.90 \pm 0.58$ & $-7.25 \pm 0.46$ & 152.22 & $<0.001$
\end{tabular}

$\begin{array}{llllll}\text { miR-144-5p } & 16.85 \pm 0.40 & 26.30 \pm 0.77 & -9.45 \pm 0.50 & 699.41 & <0.001\end{array}$

$\begin{array}{llllll}\text { miR-451a } & 13.66 \pm 0.36 & 22.89 \pm 0.51 & -9.23 \pm 0.36 & 600.49 & <0.001\end{array}$

$\begin{array}{llllll}\text { miR- } & 16.69 \pm 0.15 & 17.13 \pm 0.36 & -0.45 \pm 0.23 & 1.37 & 0.157\end{array}$

4732-3p

miR-

4732-5p

$19.50 \pm 0.46 \quad 20.87 \pm 0.05 \quad-1.37 \pm 0.27 \quad 2.58 \quad 0.025$

pri-miRNA

$10.50 \pm 0.14 \quad 15.71 \pm 1.26-5.21 \pm 0.73 \quad 37.01$

0.002

$\beta$-catenin with the IOD of 1.01, 1.00, 1.00, 0.99 and 0.99 respectively, while the IOD for control was 0.92 . In consistent, in cells over-expressing pri-miR-144/451, the expression of phosphorylated $\beta$-catenin was up-regulated (0.73 vs 0.53 ) (Fig. 4a).

miR-144-3p and miR-451 decreased the expression of total cMyc, but in miR-451a overexpressed cell total cMyc was down-regulated. miR-144-3p decreased the expression of phosphorylated c-Myc, but the cluster increased the expression of phosphorylated c-Myc (Fig. 4b).

Expression of p53 was increased in cells over-expressing miR-4732-5p and pri-miR-144/451, miR-144-3p decreased the expression of non-activated Caspase3 (Fig. 4c).

No difference of expression of PTEN and ERK1/2 were observed among cells overexpressing miRNA and miR144/451 cluster and the control cells. miR-144-3p, miR451a, miR-4732-3p and miR-144/451 cluster decreased the expression of phosphorylated ERK1/2 (Fig. 4d).

miR-144-3p, miR-4732-5p and miR-144/451 decreased the expression of MMP9 (Fig. 4e). The expression of phosphorylated cdc2 was decreased in cells over-expressing miR-144-3p and miR-144-5p but increased in cells overexpressing miR-144/451 (Fig. 4b).

\section{Discussion}

miRNA cluster refers to miRNAs encoded closely on chromosome, these miRNAs commonly share the same promoter, and exhibit the similar expression pattern and function of regulating gene expression at the level of posttranscription. It has been reported miR-144-3p and miR-451a played as the tumor suppressor in lung cancer, gastric cancer, colorectal cancer, liver cancer and many other cancers, miR-144-5p has been reported to be tumor suppressor in bladder cancer [11-22]. In consistent, in present study, we discovered miR-144-3p,
miR-144-5p, miR-451a played a tumor suppressor role in esophageal cancer.

Considering the complexity the regulation of moleculars, the function of individual miRNA may not exactly reflect real function of the cluster. The maturity of miRNA needs undergo pri-miRNA transcripts, pre-miRNA and mature miRNA in turn [23], so we established cells over-expressing miR-144/451 by over-expressing primiR-144/451. In cells overexpressing pri-miR-144/451, the expression of miR-4732-3p and miR-4732-5p almost unchanged, considering the biological function of this two miRNAs are not remarkable as the other three, we infered that miR-4732-3p and miR-4732-5p were not the real member of miR-144/451 cluster.

The function of miR-144/451 cluster were not the summation of the individual miRNA, which were not entirely consistent with the individual miRNA. miR-144-3p, miR-451a, miR-4732-3p and miR-4732-5p inhibited cell migration, miR-144-3p, miR-144-5p, miR-451a and miR4732-3p inhibited cell proliferation, however, no change of cell migration and proliferation were observed in cells over-expressing miR-144/451. The fold change in mimic transfected cell is larger than that in stable transfected cell, the complex interaction among these miRNAs may be responsible for this result.

To better understand the possible regulatory mechanism of miR-144/451, genome expression array were performed to detect the changed mRNA profiles between cells overexpressing miR-144/451 and control. The fold change of expression of mRNAs was not large in general, biological analysis suggested miR-144/451 is closely related with phosphorylation, miR-144/451 seemed mainly regulated post transcription. According to the result and the result of cell function, we selected the corresponding key proteins for verification.

Wnt signaling pathway regulates cell differentiation, proliferation, migration and many other functions, some components of Wnt signaling pathway were also implicated in other signaling pathways [24-26]. As a key component of Wnt signaling pathway, $\beta$-catenin regulates the activation of TCF/LEF, which plays important roles in transcription regulation [27]. Phosphorylated $\beta$-catenin was unstable and could be degraded by $26 \mathrm{~S}$ protease, non-phosphorylated $\beta$-catenin was not easy to be degraded which leads to the accumulation of $\beta$-catenin, thereby phosphorylation state regulates transcription regulation. Phosphorylation of Thr41, Ser37 and Ser33 is the main degradation pathway $[28,29]$. In present study, miR-144-3p, miR-144-5p and miR-451a increased the expression of phosphorylated $\beta$-catenin, these miRNAs played a synergistic effect on the inhibition of $\mathrm{Wnt} / \beta$ catenin signaling pathway. 


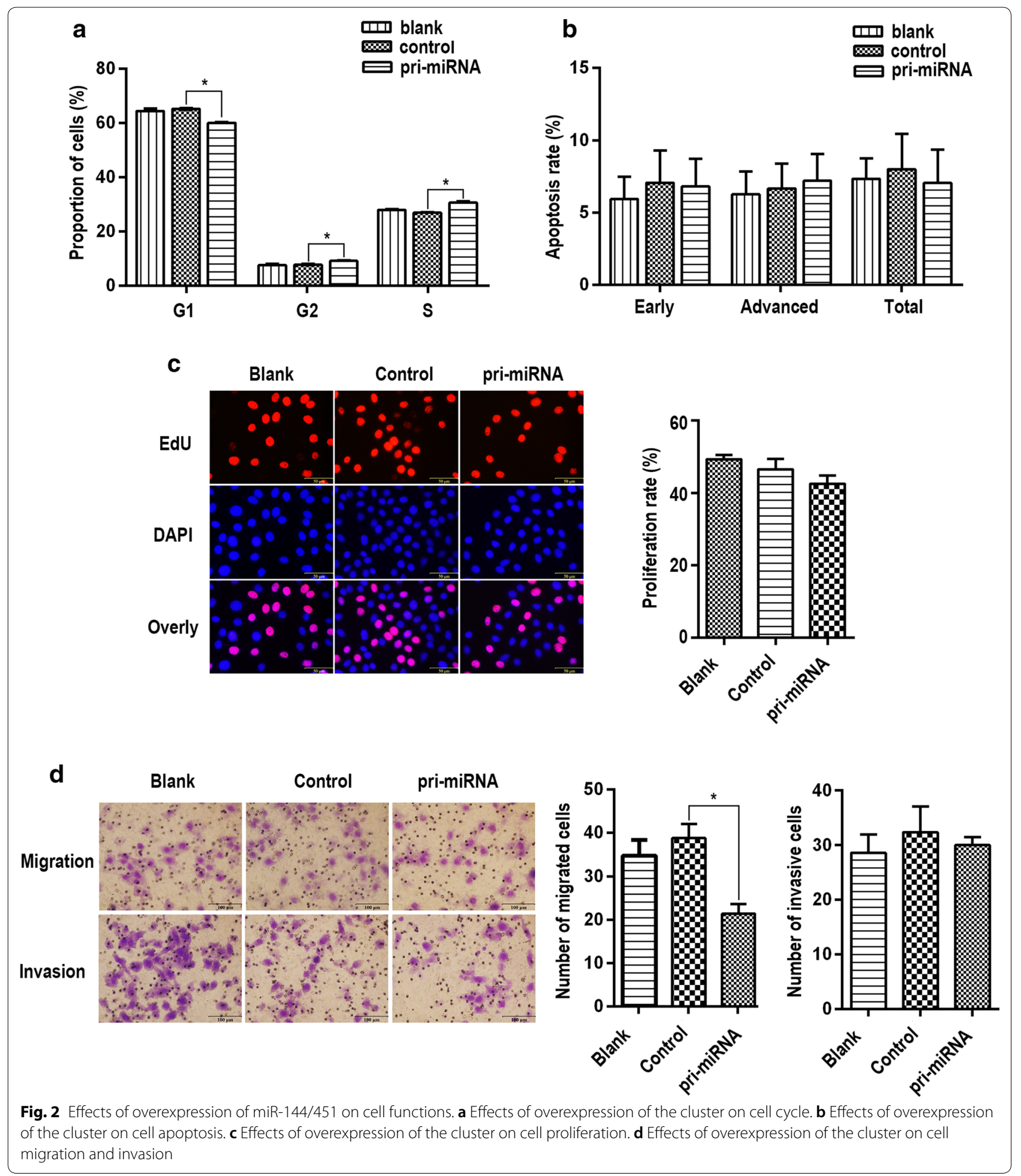

As transcription factor, c-Myc was reported to inhibit cell apoptosis and promote cell proliferation by activating key protein implicated in apoptosis and proliferation, such as RAS, RAF, BCL-2 and c-ABL [29-34].
Phosphorylation of the Ser62 Strengthened stability of c-Myc, therefore prolonged the half-life of c-Myc [35]. In this study, miR-144-3p decreased the expression of c-Myc, miR-144-5p and miR-451a increased the 


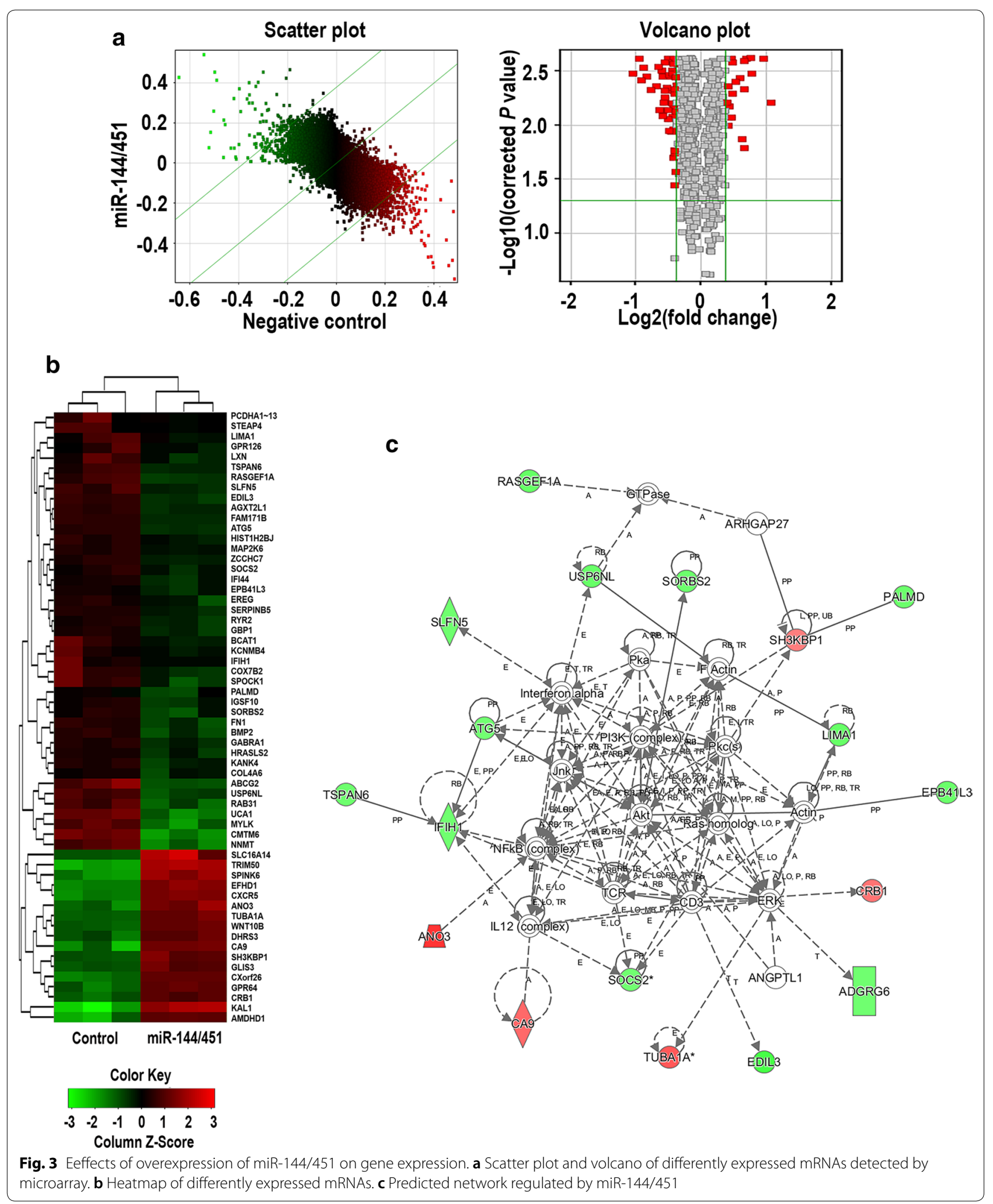


a

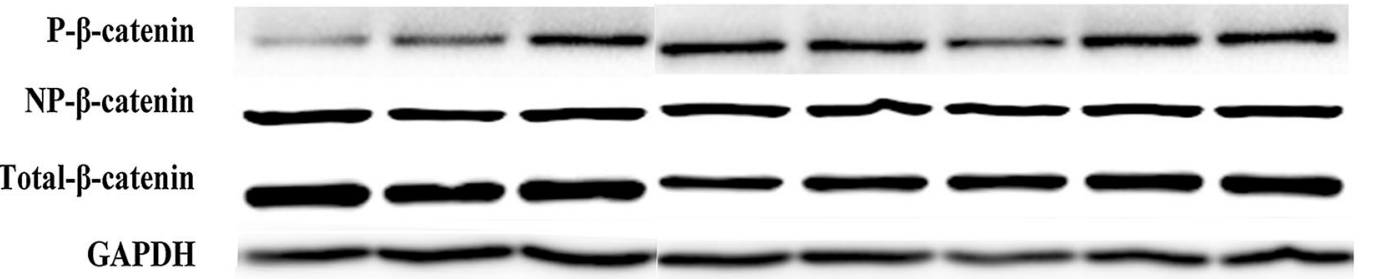

92KD

92KD

92KD
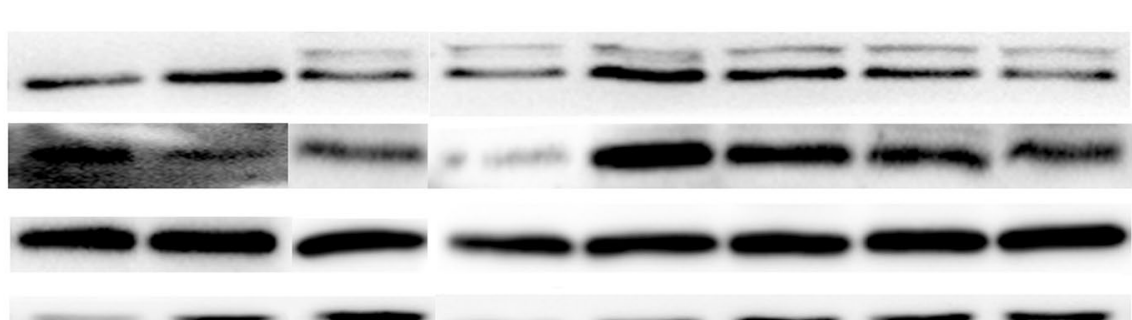

62KD

b

P-cMyc

cMyc
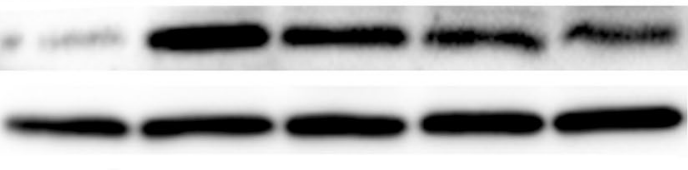

60KD

GAPDH
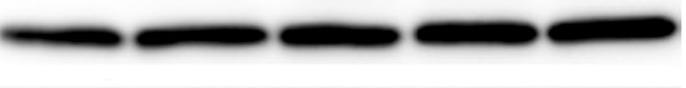

36KD

P-cdc2

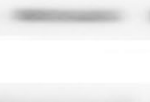

c

$$
\text { p53 }
$$
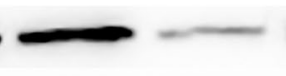

$\longrightarrow$
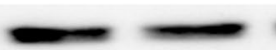

$\longrightarrow$

34KD

\section{GAPDH}

Caspase 3
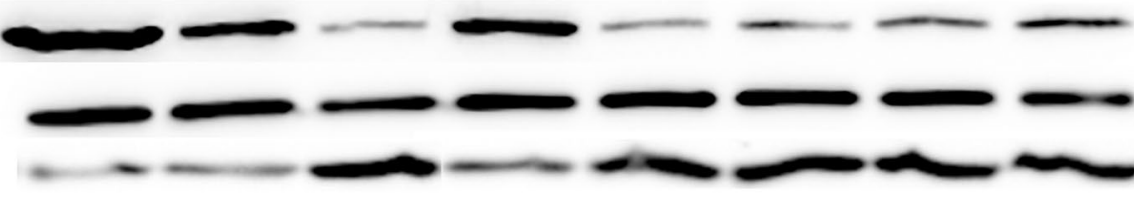

$53 \mathrm{KD}$

$36 \mathrm{KD}$

$35 \mathrm{KD}$

d
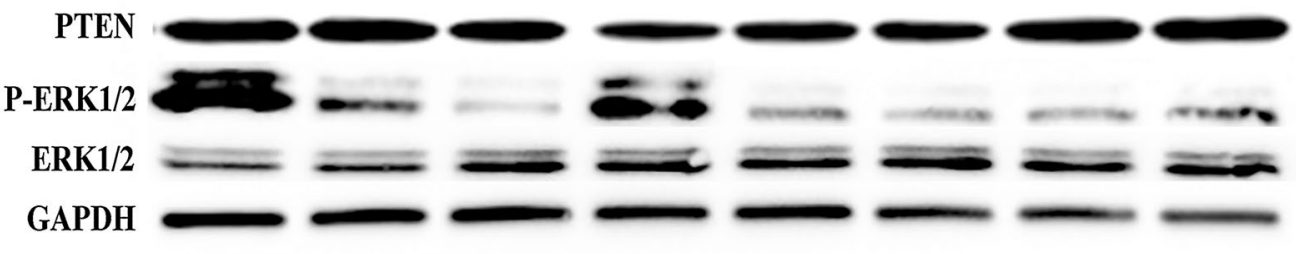

$54 \mathrm{KD}$

$42 \mathrm{KD}$

$42 \mathrm{KD}$

$36 \mathrm{KD}$

e

MMP9
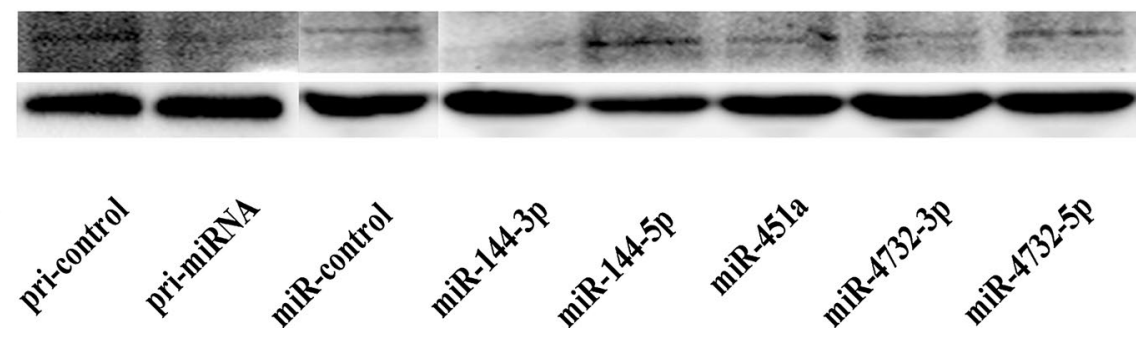

92KD

36KD

Fig. 4 Effect of miR-144/451 cluster onexpression of possible key proteins. a Expression of $\beta$-catenin. b Expression of c-Myc and phosphorylated cdc2. c Expression of MAPK/ERK pathway related proteins. $\mathbf{d}$ Expression of p53 and caspase3. e Expression of MMP9

expression of c-Myc, miR-144/451 decreased the expression of c-Myc, however the expression of phosphorylated c-Myc was down-regulated, suggesting that the low expression of c-Myc may be mainly due to the inhibition of transcriptional translation but not degradation.

MAPK/ERK signaling pathway played important roles in a variety of tumors, by regulating cyclinD1, ERK regulates the transformation of cell cycle from $\mathrm{G} 1$ to $\mathrm{S}$ phase. The signaling pathway is reported to be closely related with matrix metalloproteinase (MMPs) which is essential for cell invasion [36, 37]. As the upstream regulatory protein of MAPK/ERK signaling pathway, in this study miR144/451 have no effect on expression of PTEN [38], while the expression of phosphorylated ERK1/2 was decreased by over-expressing of miR-144/451 cluster, no change of the expression of Non- phosphorylated ERK1/2 were observed. The inhibition of MAPK/ERK signaling pathway may mainly caused by posttranscriptional 
modification of ERK1/2, miR-144-3p and miR-451a played a synergistic role in the inhibition of MAPK/ERK signaling pathway.

cdc2 plays important roles on regulating cell cycle. In advanced G2 phase, cdc2 combined with cyclinB to promote cell entry into $M$ phase [39, 40]. Dephosphorylation of Tyr15 is essential to the activation of cdc2. miR-144/451 increased the expression of p-cdc2, which was consistent with the change of cell cycle.

In this study, over-expression of miR-144/451 up-regulated the expression of p53, interestingly, in miR-144-3p, miR-144-5p and miR-451a over-expressing cells no upregulation of p53 were observed. miR-144/451 cluster is not a simple functional superposition of individual miRNAs. As key protein regulating cell apoptosis, Caspase3 exists in normal cells in the form of inactive zymogen, at early stage of apoptosis, Caspase 3 can be activated and split into $12 \mathrm{KD}$ and $17 \mathrm{KD}$ products $[41,42]$. In this study, miR-144-3p decreased the expression of 34KD Caspase3, no change in the expression of Caspase 3 were observed in stable cell over-expressing miR-144/451, which were consistent with results of cell apoptosis.

\section{Conclusion}

miR-144-451 inhibited invasion and cell cycle of EC9706. miR-144-3p prometed the apoptosis of EC9706, miR451a can lead to the arrest of G2 phase, miR-144-3, miR-451a,miR-4732-3p inhibited the migration of cell, miR-144-3p inhibited the invasion of cell, the proliferation can be inhibited by miR-144-3p, miR-144-5p, miR451a and miR-4732-3p. Wnt and MAPK/ERK/c-Myc signaling pathways were inhibited by miR-144-451 cluster, at while, miR-144/451 cluster downregulated the expression of MMP9 and upregulated the expression of $\mathrm{p}$-cdc2 which may participated in the process of cell cycle arrest and invasion inhibition.

\section{Additional files}

Additional file 1: Table S1. Abnormally expressed mRNAs in miR-144/451 overexpressing.

Additional file 2: Table S2. Upstream regulators of mRNAs abnormally expressed.

Additional file 3: Table S3. Relative expression of proteins.

\section{Abbreviations}

EdU: 5-ethynyl-2'-deoxyuridine; IPA: Ingenuity Pathway Analysis; BCA: bicinchoninic acid; MMLV: Moloney Murine Leukemia Virus; IOD: integrated option density.

\section{Authors' contributions}

ZG, RL and LY conceived and designed the experiments. ZG and HG performed the in vitro experiments, $\mathrm{PZ}$ and $\mathrm{MX}$ collected esophageal carcinoma tissues, ZG analyzed the data and wrote the paper. All authors read and approved the final manuscript.

\section{Author details}

${ }^{1}$ Key Laboratory of Environmental Medicine Engineering, Ministry of Education, School of Public Health, Southeast University, Nanjing 210009, China.

${ }^{2}$ Huzhou Center for Disease Control and Prevention, Huzhou 313000, China.

${ }^{3}$ North China Petroleum Bureau General Hospital, Renqiu 062552, China.

\section{Acknowledgements}

Not applicable.

\section{Competing interests}

The authors declare that they have no competing interests.

\section{Availability of data and materials}

Data from the cell line microarray experiments will be made available on the National Center for Biotechnology Information's gene expression omnibus, the Record Number is GSE1 18249.

\section{Consent for publication}

This manuscript is approved by all authors for publication in Cancer Cell International.

\section{Ethics approval and consent to participate}

Not applicable.

\section{Funding}

This work was supported by National Natural Science Foundation of China Grants $(81872579,81573108,81573191)$, New Century Excellent Talents in University from Ministry of Education (NCET-13-0124) and Postgraduate Research \& Practice Innovation Program of Jiangsu Province (KYCX17_0188), and Zhejiang Province Public Technology Application Research Project (No. 2016C33218).

\section{Publisher's Note}

Springer Nature remains neutral with regard to jurisdictional claims in published maps and institutional affiliations.

Received: 8 Auqust 2018 Accepted: 6 November 2018 Published online: 15 November 2018

\section{References}

1. McGuire S. World cancer report 2014. Geneva: World Health Organization, International Agency for Research on Cancer, WHO Press; 2015.

2. Torre LA, et al. Global cancer statistics, 2012. CA Cancer J Clin. 2015;65(2):87-108

3. Hammad H, Kaltenbach T, Soetikno R. Endoscopic submucosal dissection for malignant esophageal lesions. Curr Gastroenterol Rep. 2014;16(5):386.

4. Ikebe M, et al. Neoadjuvant therapy for advanced esophageal cancer: the impact on surgical management. Gen Thorac Cardiovasc Surg. 2016;64(7):386-94.

5. Bartel DP. MicroRNAs: target recognition and regulatory functions. Cell. 2009;136(2):215-33.

6. Ambros $V$. The functions of animal microRNAs. Nature. 2004;431(7006):350-5.

7. Esquela-Kerscher A, Slack FJ. Oncomirs-microRNAs with a role in cancer. Nat Rev Cancer. 2006;6(4):259-69.

8. Yu J, et al. Human microRNA clusters: genomic organization and expression profile in leukemia cell lines. Biochem Biophys Res Commun. 2006;349(1):59-68.

9. Wystub K, et al. miR-1/133a clusters cooperatively specify the cardiomyogenic lineage by adjustment of myocardin levels during embryonic heart development. PLoS Genetics. 2013;9(9):e1003793.

10. Gao Z, et al. Possible tumor suppressive role of the miR-144/451 cluster in esophageal carcinoma as determined by principal component regression analysis. Mol Med Rep. 2016;14(4):3805-13. 
11. Zha W, et al. Roles of mir-144-ZFX pathway in growth regulation of nonsmall-cell lung cancer. PLoS ONE. 2013;8(9):e74175.

12. Liu J, et al. MicroRNA-144 inhibits the metastasis of gastric cancer by targeting MET expression. J Exp Clin Cancer Res. 2015;34:9.

13. Xiao R, Li C, Chai B. miRNA-144 suppresses proliferation and migration of colorectal cancer cells through GSPT1. Biomed Pharmacother. 2015;74:138-44.

14. Ma Y, et al. MicroRNA-144 suppresses tumorigenesis of hepatocellular carcinoma by targeting AKT3. Mol Med Rep. 2015;11(2):1378-83.

15. Matsushita R, et al. Tumour-suppressive microRNA-144-5p directly targets CCNE1/2 as potential prognostic markers in bladder cancer. Br J Cancer. 2015;113(2):282-9.

16. Turczynska KM, et al. Stretch-sensitive down-regulation of the mir144/451 cluster in vascular smooth muscle and its role in AMP-activated protein kinase signaling. PLOS ONE. 2013;8(5):e65135.

17. Wang $R$, et al. MicroRNA-451 functions as a tumor suppressor in human non-small cell lung cancer by targeting ras-related protein 14 (RAB14). Oncogene. 2011;30(23):2644-58.

18. Babapoor S, et al. A novel miR-451a isomiR, associated with amelanotypic phenotype, acts as a tumor suppressor in melanoma by retarding cell migration and invasion. PLoS ONE. 2014;9(9):e107502.

19. Liu ZR, et al. miR-451a inhibited cell proliferation and enhanced tamoxifen sensitive in breast cancer via macrophage migration inhibitory factor. Biomed Res Int. 2015;2015:12.

20. Li H-P, et al. miR-451 inhibits cell proliferation in human hepatocellular carcinoma through direct suppression of IKK-beta. Carcinogenesis. 2013;34(11):2443-51.

21. Bergamaschi A, Katzenellenbogen BS. Tamoxifen downregulation of miR451 increases 14-3-3 zeta and promotes breast cancer cell survival and endocrine resistance. Oncogene. 2012;31(1):39-47.

22. Yuan J, et al. The expression and function of miRNA-451 in osteosarcoma. Med Oncol. 2015;32(1):324.

23. Lee $Y$, et al. MicroRNA genes are transcribed by RNA polymerase II. EMBO J. 2004;23(20):4051-60.

24. Angbohang A, et al. Downregulation of the canonical WNT signaling pathway by TGF beta 1 inhibits photoreceptor differentiation of adult human Muller glia with stem cell characteristics. Stem Cells Dev. 2016;25(1):1-12.

25. Hamada F. Wnt signaling and cancer. Kaibogaku zasshi J Anat. 2009;84(4):111-2

26. Clevers H. Wnt/beta-catenin signaling in development and disease. Cell. 2006;127(3):469-80.
27. Kikuchi A, Kishida S, Yamamoto H. Regulation of Wnt signaling by proteinprotein interaction and post-translational modifications. Exp Mol Med. 2006;38(1):1-10.

28. Liu CM, et al. Control of beta-catenin phosphorylation/degradation by a dual-kinase mechanism. Cell. 2002;108(6):837-47.

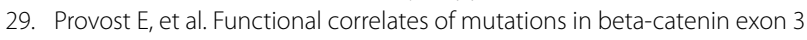
phosphorylation sites. J Biol Chem. 2003;278(34):31781-9.

30. $\mathrm{Xu} \mathrm{JH}$, et al. Catenin regulates C-Myc and CDKN1A expression in breast cancer cells. Mol Carcinog. 2016;55(5):431-9.

31. Ponzielli R, et al. Cancer therapeutics: targeting the dark side of Myc. Eur J Cancer. 2005;41(16):2485-501.

32. Oster SK, et al. The myc oncogene: MarvelousIY Complex. Adv Cancer Res. 2002;84:81-154.

33. Nilsson JA, Cleveland JL. Myc pathways provoking cell suicide and cancer. Oncogene. 2003;22(56):9007-21.

34. Pelengaris S, Khan M, Evan Gl. Suppression of Myc-induced apoptosis in beta cells exposes multiple oncogenic properties of Myc and triggers carcinogenic progression. Cell. 2002;109(3):321-34

35. Wang W, et al. SCP1 regulates c-Myc stability and functions through dephosphorylating c-Myc Ser62. Oncogene. 2016;35(4):491-500.

36. Yang C-Q, et al. MCP-1 stimulates MMP-9 expression via ERK $1 / 2$ and p38 MAPK signaling pathways in human aortic smooth muscle cells. Cell Physiol Biochem. 2014;34(2):266-76.

37. Chang MC, et al. Mesothelin enhances invasion of ovarian cancer by inducing MMP-7 through MAPK/ERK and JNK pathways. Biochem J. 2012;442:293-302.

38. Chetram MA, Hinton CV. PTEN regulation of ERK1/2 signaling in cancer. J Recept Signal Transduct Res. 2012;32(4):190-5.

39. Aleem E, Kiyokawa H, Kaldis P. Cdc2-cyclin E complexes regulate the G1/S phase transition. Nat Cell Biol. 2005;7(8):831-U93.

40. Sun W-J, et al. Romidepsin induces G2/M phase arrest via Erk/cdc25C cdc2/cyclinB pathway and apoptosis induction through JNK/c-Jun/ caspase 3 pathway in hepatocellular carcinoma cells. Biochem Pharmacol. 2017;127:90-100

41. Zheng TS, et al. Caspase-3 controls both cytoplasmic and nuclear events associated with Fas-mediated apoptosis in vivo. Proc Natl Acad Sci USA. 1998;95(23):13618-23.

42. Olie RA, et al. A novel antisense oligonucleotide targeting survivin expression induces apoptosis and sensitizes lung cancer cells to chemotherapy. Can Res. 2000;60(11):2805-9.
Ready to submit your research? Choose BMC and benefit from:

- fast, convenient online submission

- thorough peer review by experienced researchers in your field

- rapid publication on acceptance

- support for research data, including large and complex data types

- gold Open Access which fosters wider collaboration and increased citations

- maximum visibility for your research: over 100M website views per year

At $\mathrm{BMC}$, research is always in progress.

Learn more biomedcentral.com/submissions 